\title{
Theoretical Reflectance Spectra of Earth-like Planets through Their Evolutions: Impact of Clouds on the Detectability of Oxygen, Water, and Methane with Future Direct Imaging Missions
}

\author{
Yui Kawashima ${ }^{1,2,3}$ (D) and Sarah Rugheimer ${ }^{4,5}$ \\ ${ }^{1}$ SRON Netherlands Institute for Space Research, Sorbonnelaan 2, 3584 CA Utrecht, The Netherlands; y.kawashima@sron.nl \\ ${ }^{2}$ Earth-Life Science Institute, Tokyo Institute of Technology 2-12-1-IE-1 Ookayama, Meguro-ku, Tokyo 152-8550, Japan \\ ${ }^{3}$ Department of Earth and Planetary Science, Graduate School of Science, The University of Tokyo, 7-3-1 Hongo, Bunkyo-ku, Tokyo 113-0033, Japan \\ ${ }^{4}$ Atmospheric, Oceanic, and Planetary Physics Department, University of Oxford, Clarendon Laboratory, Parks Road, Oxford OX1 3PU, UK \\ ${ }^{5}$ School of Earth and Environmental Science, University of St Andrews, Irvine Building, St. Andrews KY16 9AL, UK \\ Received 2018 November 30; revised 2019 March 26; accepted 2019 March 28; published 2019 May 6
}

\begin{abstract}
In the near-future, atmospheric characterization of Earth-like planets in the habitable zone will become possible via reflectance spectroscopy with future telescopes such as the proposed LUVOIR and HabEx missions. While previous studies have considered the effect of clouds on the reflectance spectra of Earth-like planets, the molecular detectability considering a wide range of cloud properties has not been previously explored in detail. In this study, we explore the effect of cloud altitude and coverage on the reflectance spectra of Earth-like planets at different geological epochs and examine the detectability of $\mathrm{O}_{2}, \mathrm{H}_{2} \mathrm{O}$, and $\mathrm{CH}_{4}$ with test parameters for the future mission concept, LUVOIR, using a coronagraph noise simulator previously designed for WFIRST-AFTA. Considering an Earth-like planet located at $5 \mathrm{pc}$ away, we have found that for the proposed LUVOIR telescope, the detection of the $\mathrm{O}_{2} A$-band feature $(0.76 \mu \mathrm{m})$ will take approximately 100,30 , and $10 \mathrm{hr}$ for the majority of the cloud parameter space modeled for the atmospheres with $10 \%, 50 \%$, and $100 \%$ of modern Earth $\mathrm{O}_{2}$ abundances, respectively. In particular, for the case of $\gtrsim 50 \%$ of modern Earth $\mathrm{O}_{2}$ abundance, the feature will be detectable with an integration time $\lesssim 10 \mathrm{hr}$ as long as there are lower-altitude $(\lesssim 8 \mathrm{~km})$ clouds with a global coverage of $\gtrsim 20 \%$. For the $1 \%$ of the modern Earth $\mathrm{O}_{2}$ abundance case, however, it will take more than $100 \mathrm{hr}$ for all the cloud parameters we modeled.
\end{abstract}

Key words: planets and satellites: atmospheres - planets and satellites: detection - planets and satellites: terrestrial planets

\section{Introduction}

With recent advances in observational techniques, more than 3000 exoplanets have been reported so far $^{6}$ with many more nearby habitable exoplanets expected to be discovered by the Transiting Exoplanet Survey Satellite (TESS). Already, some rocky planets have been found in habitable zones (HZs) of their host stars such as Proxima Centauri b, TRAPPIST-1 (e, f, and g), and LHS 1140b (Anglada-Escudé et al. 2016; Dittmann et al. 2017; Gillon et al. 2017). The next step will be to characterize the atmospheres of these planets. For characterization of planets in the HZs, reflectance spectroscopy is most suitable for the planets around F-, G-, and K-type stars because of the larger angular separation of the HZs from those host stars. Transmission spectroscopy suits the characterization of the planets in the HZs around M dwarfs because of their larger transit probabilities and larger planet-to-star radius ratios.

The first telescopes capable of characterizing rocky habitable planet's atmospheres will be the James Webb Space Telescope (JWST; launching in 2021) and through high-resolution spectroscopy with large ground-based telescopes coming online in the 2020s such as the Extremely Large Telescope (ELT; $39 \mathrm{~m}$ ). However, these missions will only be able to characterize a handful of habitable worlds. As such, future mission concepts like the Large UV/Optical/IR Surveyor (LUVOIR) and the Habitable Exoplanet Observatory (HabEx) are being proposed that would be able to detect and characterize statistically meaningful samples (see Stark et al. 2014, 2015). Compared

\footnotetext{
6 http://exoplanets.org
}

to JWST with a diameter of $6.5 \mathrm{~m}$ and a wavelength coverage of $0.6-28.5 \mu \mathrm{m}$, LUVOIR is proposed to have a much larger diameter of 15 or $8 \mathrm{~m}$ and would probe a shorter wavelength range of $0.1-2.5 \mu \mathrm{m}$ and a coronagraph with the possibility of a starshade. ${ }^{7}$ HabEx, a $4 \mathrm{~m}$ telescope, is proposed to have a starshade and a coronagraph and likewise will probe a shorter wavelength range than JWST, $0.2-1.8 \mu \mathrm{m} .{ }^{8}$ LUVOIR and $H a b E x$ will be suitable for the detection and characterization of planets in the HZs around F-, G-, and K-type stars via reflectance spectroscopy, while JWST is best suited for transiting planets in the HZs around M dwarfs.

Among the several proposed biosignature gases, the existence of molecular oxygen in the atmosphere has been long considered as one of the most promising biosignature candidates for Earthlike planets (see reviews by Meadows 2017; Meadows et al. 2018, and references therein). Although several abiotic sources of $\mathrm{O}_{2}$ have been proposed so far $(\mathrm{Hu}$ et al. 2012; DomagalGoldman et al. 2014; Ramirez \& Kaltenegger 2014; Tian et al. 2014; Wordsworth \& Pierrehumbert 2014; Gao et al. 2015; Harman et al. 2015; Luger \& Barnes 2015; Narita et al. 2015), the simultaneous detection of large abundances of $\mathrm{O}_{2}$ or its photochemical byproduct $\mathrm{O}_{3}$ in combination with a reducing gaseous species such as $\mathrm{CH}_{4}$ is still considered as the most robust biosignature. This is because as reduced and oxidizing gases react rapidly with each other, such a detection assures a large flux of $\mathrm{O}_{2}$ and $\mathrm{CH}_{4}$ from the surface, and therefore are likely biotic in origin (Lederberg 1965; Lovelock 1965;

\footnotetext{
https://asd.gsfc.nasa.gov/luvoir/

8 https://www.jpl.nasa.gov/habex/
} 
Sagan et al. 1993). Also, $\mathrm{H}_{2} \mathrm{O}$, while not a biosignature, is a useful indicator of habitability.

Earth's atmosphere has been very different in its history, representing a variety of possible terrestrial atmospheres (Kaltenegger et al. 2007; Rugheimer \& Kaltenegger 2018). In addition, we expect to find atmospheric compositions far beyond what we have seen in the Earth's history or in our solar system bodies as the detection of hot Jupiters and mini-Neptunes have already shown. However, it is not unreasonable to search for $\mathrm{O}_{2}$ as the building blocks of the oxygenic photosynthesis $\left(\mathrm{H}_{2} \mathrm{O}, \mathrm{CO}_{2}\right.$, and photons) are abundant in the universe. Their widespread availability in part has made oxygenic photosynthesis the most successful biomass building strategy on the Earth. While $\mathrm{O}_{2}$ abundance in the atmospheres of habitable planets could be much less, it is likely not much more on a habitable planet with vegetation due to widespread fires if $\mathrm{O}_{2}$ increases above $25 \%-$ $35 \%$ of the atmosphere due to widespread fires (Watson et al. 1978; Scott \& Glasspool 2006). Also, in Earth's history, $\mathrm{O}_{2}$ has not exceeded $\sim 30 \%-35 \%$ (Kump 2008; Lyons et al. 2014).

The observation of flat or featureless spectra for a number of exoplanets has demonstrated the commonality of clouds and hazes (e.g., Kreidberg et al. 2014; Sing et al. 2016). By absorbing and scattering the light, the existence of clouds and hazes can significantly impact the spectrum of the planet (e.g., Kawashima \& Ikoma 2018, 2019; Kawashima et al. 2019). On Earth, the high albedo of water and ice clouds compared to that of the surface can deepen molecular absorption features, while also obscuring features depending on the cloud properties (the altitude of the cloud layer and its fractional coverage; e.g., Tinetti et al. 2006a, 2006b; Kaltenegger et al. 2007; Kitzmann et al. 2011; Rugheimer et al. 2013).

Previous studies have modeled the reflectance spectra of modern Earth-like planets considering the effect of clouds in the atmospheres (e.g., Des Marais et al. 2002; Tinetti et al. 2006a, 2006b; Kitzmann et al. 2011, 2013; Robinson et al. 2011; Rugheimer et al. 2013, 2015a; Sanromá et al. 2013, 2014; Feng et al. 2018; Wang et al. 2018). In addition to modern Earthlike planets, Kaltenegger et al. (2007) and Rugheimer \& Kaltenegger (2018) modeled the reflectance spectra of planets similar to the Earth at earlier geological epochs orbiting around Sun-like stars, and those around F, G, K, and M stars, respectively. While most of the above studies considered clouds with altitudes and global average coverage similar to the modern Earth, the cloud properties in other Earth-like planets are unknown and will be likely different from those of the modern Earth. The detectability of molecular features considering such a wide range of cloud properties has not been explored in detail.

In this study, we explore the effect of water and ice cloud properties, namely, the altitude and its coverage, on the reflectance spectra of Earth-like planets around Sun-like stars at different geological epochs and examine the detectability of astrobiologically interesting gaseous molecules in the visible and near-infrared spectrum, namely, $\mathrm{O}_{2}, \mathrm{H}_{2} \mathrm{O}$, and $\mathrm{CH}_{4}$, with test parameters for the future mission concept, LUVOIR, using a scaled the Wide Field Infrared Survey Telescope-AstropysicsFocused Telescope Assets (WFIRST-AFTA) coronagraph noise simulator (Robinson et al. 2016).

The rest of this paper is organized as follows. In Section 2, we describe our model. In Section 3, we show the results of reflectance spectrum models of Earth-like planets at different geological epochs and systematically explore the effect of the cloud properties. In Section 4, we report the detectability of $\mathrm{O}_{2}$,
$\mathrm{H}_{2} \mathrm{O}$, and $\mathrm{CH}_{4}$ in these atmospheres using potential parameters for the future mission concept, LUVOIR. Then in Sections 5 and 6 , we conclude this paper by discussing our treatment of clouds and summarizing the results.

\section{Methods}

We simulate the reflectance spectra considering the planets with the same mass, radius, and semimajor axis as the Earth orbiting the star with the same properties as the Sun at different geological epochs. Out of four geological epochs considered in Rugheimer \& Kaltenegger (2018), we consider the three epochs when the Earth has had an active biosphere and oxygenic photosynthesis, $2.0 \mathrm{Ga}, 0.8 \mathrm{Ga}$, and the present. $2.0 \mathrm{Ga}$ corresponds to the time after the Great Oxidation Event (GOE) of $\sim 2.33 \mathrm{Ga}$ (e.g., Luo et al. 2016) when $\mathrm{O}_{2}$ started to build up in the atmosphere and $0.8 \mathrm{Ga}$ corresponds to the time when multicellular life started to proliferate after the Neoproterozoic Oxidation Event (NOE).

\subsection{Reflectance Spectrum Model}

To simulate reflectance spectra of Earth-like planets, we use a line-by-line radiative transfer model (Traub \& Stier 1976; Kaltenegger \& Traub 2009; Rugheimer \& Kaltenegger 2018). We calculate the spectra with a wavenumber grid width of $0.01 \mathrm{~cm}^{-1}$. We use the temperature-pressure profile and distribution of gaseous species of Rugheimer et al. (2015b) for Earth-like atmospheres at the three geological epochs as inputs to the radiative transfer model, which are shown in Figure 1. Those results were calculated with a 1D climate model (Kasting \& Ackerman 1986; Pavlov et al. 2000; HaqqMisra et al. 2008) and a 1D photochemistry code (Pavlov \& Kasting 2002; Segura et al. 2005, 2007).

Note that the temperature and abundances for the two earlier epochs are not well constrained and lie within an extremely broad range of possible values. We tabulate the geological constraints on the past $\mathrm{O}_{2}$ abundance for each geological epoch in Table 1. As for $\mathrm{H}_{2} \mathrm{O}$, its abundance in the atmosphere is determined by evaporation and thus surface temperature. However, considering that the temperature oscillation occurred during the cooler period within the huge temporal range, it might be lower than what we assume here.

For $\mathrm{CH}_{4}$, its past abundance in the atmosphere is not currently constrained by geological records. The photochemical model of Pavlov et al. (2003) predicted a concentration of 100-300 ppm in the Proterozoic $(0.75-2.3 \mathrm{Ga})$ atmosphere in order to maintain warm climate against the faint early Sun. The biogeochemical model of Claire et al. (2006) derived an analytical solution of $\mathrm{CH}_{4}$ abundance as a function of uncertain parameters such as rate coefficient for a $\mathrm{CH}_{4}$ destruction by $\mathrm{O}_{2}$, surface biogenic flux of $\mathrm{CH}_{4}$, and the $\mathrm{O}_{2}$ abundance. Their reference model predicted its abundance ranges from 10 to $100 \mathrm{ppm}$ after GOE at $2.3 \mathrm{Ga}$. In absence of robust geological paleosol records, we have adopted optimistic $\mathrm{CH}_{4}$ levels in the lowest $\mathrm{O}_{2}$ case. Future work will be needed to constrain $\mathrm{CH}_{4}$ abundance in Earth's history.

As for clouds, we assume water (cumulus) clouds for temperature above $230 \mathrm{~K}$ and ice (cirrus) clouds for that below $230 \mathrm{~K}$, following Zsom et al. (2012). We insert continuumabsorbing/emitting layers similar to some previous works (Des Marais et al. 2002; Kaltenegger et al. 2007; Rugheimer et al. 2013, 2015a; Rugheimer \& Kaltenegger 2018). While planets with a surface ocean, an active hydrological cycle, and 
Temperature $[\mathrm{K}]$

Temperature $[\mathrm{K}]$

Temperature $[\mathrm{K}]$

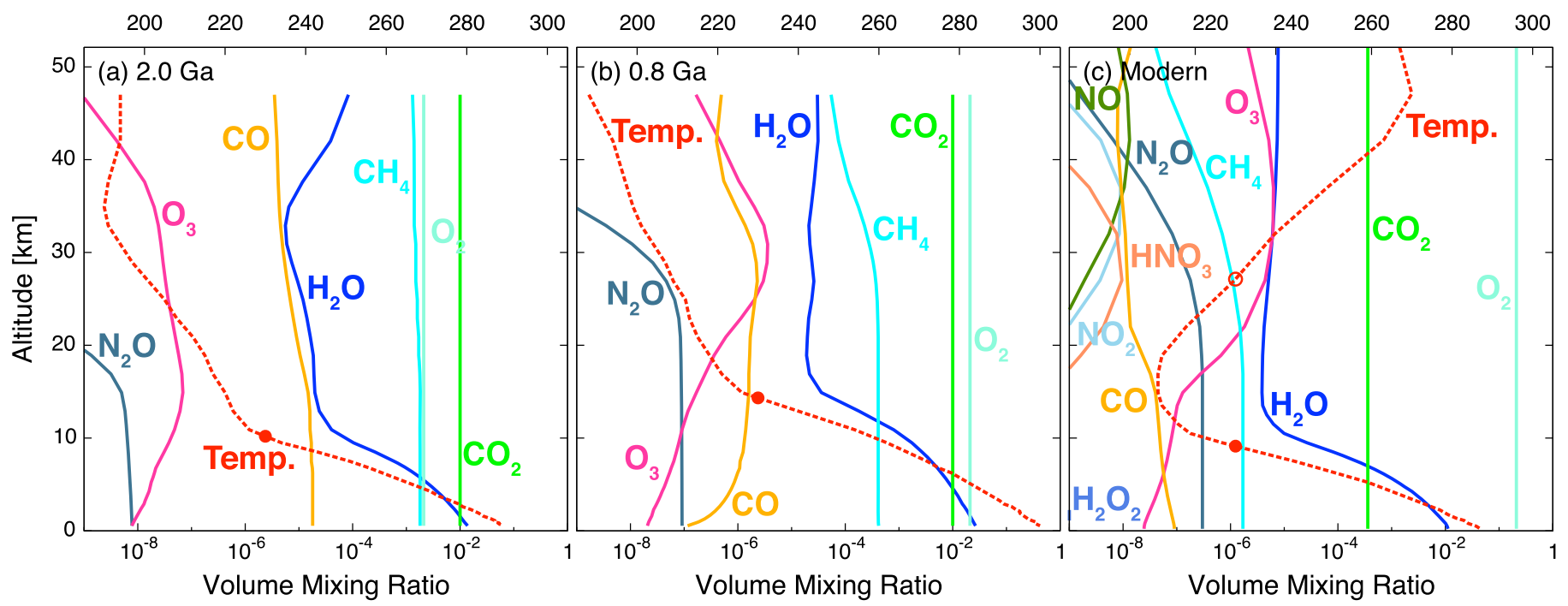

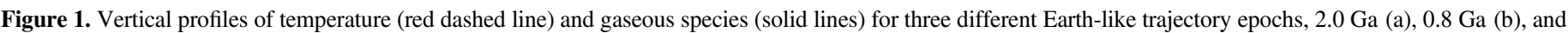

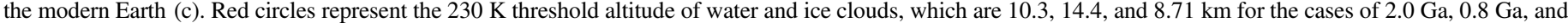
the modern Earth, respectively. Note that most abundant species $\mathrm{N}_{2}$ is not shown.

Table 1

Geological Constraints on the Past $\mathrm{O}_{2}$ Abundances

\begin{tabular}{lll}
\hline \hline Epoch [Ga.] & Concentration & Reference \\
\hline $2.45-0.42$ & $0.01-0.4$ PAL & Kump (2008) and references therein \\
$2.1-0.8$ & $10^{-4}-0.1$ PAL & Lyons et al. (2014) and references therein \\
$1.8-0.8$ & $<0.001$ PAL & Planavsky et al. (2014) \\
$0.42-0.0$ & $0.6-1.6$ PAL & Kump (2008) and references therein \\
\hline
\end{tabular}

Note. PAL stands for the present atmospheric level.

abundant water vapor have abundant clouds, dry habitable planets, which have been proposed to extend the $\mathrm{HZ}$ inward (e.g., Abe et al. 2005, 2011; Zsom et al. 2013; Kodama et al. 2015), have fewer clouds (e.g., Kodama et al. 2018). However, as the cloud properties in exoplanet contain large uncertainty, we simply vary the altitude of the cloud layer and its coverage systematically to explore the effect of these cloud properties on reflectance spectra of Earth-like exoplanets.

We assume surface compositions following Rugheimer \& Kaltenegger (2018): the surface consists of $70 \%$ ocean, $2 \%$ coast, and $28 \%$ land for all the epochs considered. For 2.0 and $0.8 \mathrm{Ga}$ cases, the land is composed of $35 \%$ basalt, $40 \%$ granite, $15 \%$ snow, and $10 \%$ sand, while $30 \%$ grass, $30 \%$ trees, $9 \%$ granite, $9 \%$ basalt, $15 \%$ snow, and $7 \%$ sand for modern case. We take reflectivity data for clouds and surface compositions from the ASTER Spectral Library ${ }^{9}$ (Baldridge et al. 2009) and the USGS Spectral Library ${ }^{10}$ (Kokaly et al. 2017). We adopt the average planet phase angle of $\frac{\pi}{2}$ (i.e., quadrature). For the input stellar spectra of the Sun at each epoch, we use a solar evolution model (Claire et al. 2012).

\subsection{LUVOIR Coronagraph Noise Simulator}

We calculate the impact of noise on the detection of spectral features considering the Earth-like planet located at 5 pc away

\footnotetext{
9 http://speclib.jpl.nasa.gov

10 http://speclab.cr.usgs.gov/spectral-lib.html
}

Table 2

Values of Parameters Used in This Study

\begin{tabular}{lcc}
\hline \hline Description & Value & Reference \\
\hline Distance to observed star-planet system & $5 \mathrm{pc}$ & $\ldots$ \\
Planetary radius & $1 R_{\oplus}$ & $\ldots$ \\
Planet-star distance & $1 \mathrm{au}$ & $\ldots$ \\
Planet phase angle & $90^{\circ}$ & $\ldots$ \\
Number of exodis in exoplanetary disk & 1 & $\ldots$ \\
Coronagraph design contrast & $10^{-10}$ & $\ldots$ \\
Telescope diameter & $10 \mathrm{~m}$ & $\ldots$ \\
Instrument spectral resolution & 140 & LUVOIR interim report $^{\mathrm{a}}$ \\
Telescope and instrument throughput & 0.20 & $\ldots$ \\
Coronagraph inner working angle $[\lambda / D]$ & 3.5 & LUVOIR interim report $^{\mathrm{a}}$ \\
Coronagraph outer working angle $[\lambda / D]$ & 64.0 & LUVOIR $_{\text {interim report }}$ \\
Width of photometric aperture $[\lambda / D]$ & 1.5 & $\ldots$ \\
\hline
\end{tabular}

Note.

a https://asd.gsfc.nasa.gov/luvoir/

from the Earth. For this purpose, we use the instrument noise model from Robinson et al. (2016) originally developed for WFIRST-AFTA. We have modified this noise calculator to match the potential LUVOIR values. While two plans have been proposed for the telescope diameter of LUVOIR, $15 \mathrm{~m}$ and $8 \mathrm{~m}$, in this study, we use the value of $10 \mathrm{~m}$ as an example. Considering the visible channel of the ECLIPS instrument, we take its value for the instrument spectral resolution and coronagraph inner and outer working angles from Table 9.2 of the LUVOIR interim report (see footnote 7). All the input values we use are listed in Table 2. Also, while the original noise model assumed the blackbody for the stellar spectrum, we use the solar spectrum evolution model used in the spectrum calculations as the input (Claire et al. 2012).

Following Robinson et al. (2016), we explore the integration time required to detect a molecular feature by defining it as the time to achieve $\mathrm{S} / \mathrm{N}=5$. We define the signal as the difference between the spectra calculated with and without the specific molecular absorption, while Robinson et al. (2016) 


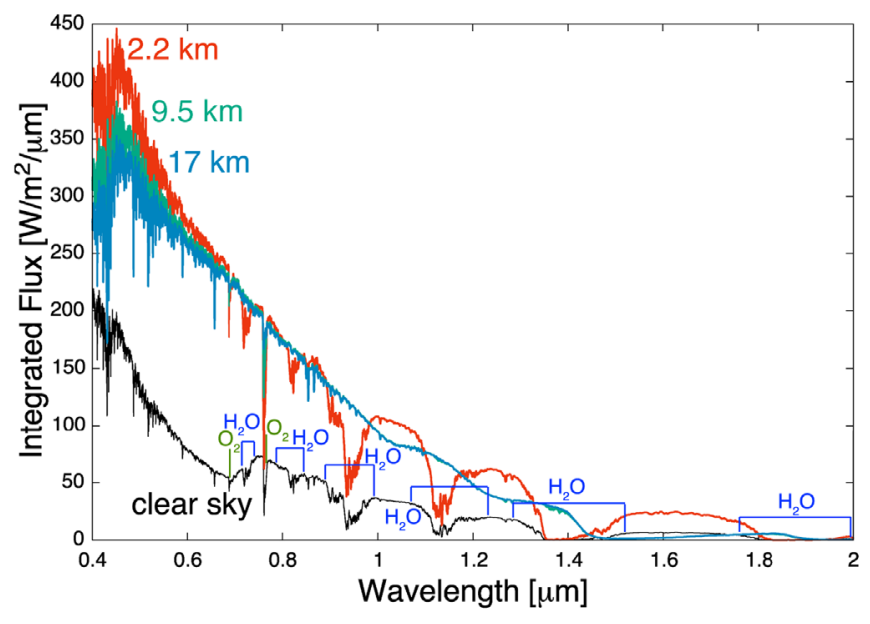

Figure 2. Reflective spectra for a modern Earth-like atmosphere with $100 \%$ cloud coverage at three different altitudes, $17 \mathrm{~km}$ (blue line), $9.5 \mathrm{~km}$ (green line), and $2.2 \mathrm{~km}$ (red line). Water clouds are assumed for the $2.2 \mathrm{~km}$ case, while ice clouds for the 17 and $9.5 \mathrm{~km}$ cases. A clear sky atmosphere is plotted in a black line for reference. Note that the spectral models are smoothed for clarity by averaging over the wavenumber range of $20.1 \mathrm{~cm}^{-1}$ at each outputted wavenumber point with a grid of $0.1 \mathrm{~cm}^{-1}$. We use the same smoothing method for the results of spectrum models hereafter.

defined it as the deviation from a flat continuum; we substitute the photon count rate for the case of the spectrum calculated without considering the absorption of a certain molecule for the continuum count rate in Equation (7) of Robinson et al. (2016). The model selects a wavelength element within a specific wavelength range from a given instrument spectral resolution. We will mention the wavelength range we adopt for each molecular absorption feature in Section 4. Note that in order to recover molecular abundances, a measurement of the flux at the bottom of the absorption features is important.

\section{Results: Influence of Clouds on Spectra of Earth-like Planets}

In this section, we systematically explore the effect of cloud properties, namely, the altitude of the cloud layer (Section 3.1) and its coverage (Section 3.2) on reflectance spectra of an Earth-like planet. Then in Section 3.3, we compare the spectrum models of the Earth-like planets at different geological epochs, focusing on the $\mathrm{O}_{2} A$-band feature as $\mathrm{O}_{2}$ has long been considered as a key target molecule for future missions.

\subsection{Altitude of Cloud Layer}

Figure 2 shows spectral models for an atmosphere with $100 \%$ cloud coverage at three different altitudes, $17 \mathrm{~km}$ (blue line), $9.5 \mathrm{~km}$ (green line), and $2.2 \mathrm{~km}$ (red line). Water clouds are assumed for the $2.2 \mathrm{~km}$ case, while ice clouds for the 17 and $9.5 \mathrm{~km}$ cases. A clear sky atmosphere is also plotted (black) for reference. One finds that in the spectrum of a clear sky atmosphere, most of the molecular absorption features come from $\mathrm{H}_{2} \mathrm{O}$, which are located at $0.71-0.74,0.80-0.84$, $0.90-0.98,1.1-1.2,1.3-1.5$, and $1.8-2.0 \mu \mathrm{m}$, while the distinct $\mathrm{O}_{2} A$-band feature exists at $0.76 \mu \mathrm{m}$ along with the smaller $\mathrm{O}_{2}$ $B$-band feature at $0.69 \mu \mathrm{m}$.

Clouds increase the flux because of their high albedo. At relatively short wavelengths $(\lesssim 0.9 \mu \mathrm{m})$, where the atmosphere is relatively optically thick and the optical properties of water and ice clouds are almost similar, the lower the altitude of the cloud layer is, the larger the overall (continuum) flux becomes. This behavior is due to the increased Rayleigh scattering of molecules above the cloud layer in the lower atmosphere. For the lower-altitude clouds, the absorption feature is deeper, and the flux is lower in the core of the line. This is because there is a larger column-integrated concentration of the species above the cloud layer (see also Tinetti et al. 2006a, 2006b; Kitzmann et al. 2011).

In contrast, at relatively long wavelengths $(\gtrsim 0.9 \mu \mathrm{m})$, where the atmosphere is optically thinner, the features are created mostly by clouds, while the molecular absorption also contributes for the lower-altitude cloud case of $2.2 \mathrm{~km}$. Note that water clouds have absorption at a similar wavelength region to gaseous water. For the higher-altitude ice cloud cases of 17 and $9.5 \mathrm{~km}$, due to the negligible column-integrated concentration of the species above the cloud layer for the both cases, the spectra are similar and completely characterized by less reflective optical properties of ice clouds.

The left panel of Figure 3 is the zoomed-in view of Figure 2 around the $\mathrm{O}_{2} A$-band feature. Note that the difference of the reflectivity of water and ice clouds is little in this wavelength region. The flux at the peak of the absorption feature is smaller for the lower cloud layer, while that at the continuum is larger as noted above. We show relative reflectivity in the right panel of Figure 3 calculated by normalizing the flux with the maximum flux between the wavelength range of $0.75-0.78 \mu \mathrm{m}$. For the lower-altitude clouds, the relative reflectivity of the feature becomes deeper due to the larger absorption at the core of the feature and increased Rayleigh scattering at the continuum.

\subsection{Cloud Coverage}

Next, we examine the dependence of the fractional cloud coverage on the spectra. The panel (a) of Figure 4 shows the Earth-like spectra with ice cloud layers of $17 \mathrm{~km}$ altitude, while panel (b) shows those with $2.2 \mathrm{~km}$ water cloud layers, for $0 \%$ (black), 50\% (blue), and 100\% (red) cloud coverage. Again, the difference of the reflectivity of water and ice clouds is little in this wavelength region. For the $17 \mathrm{~km}$ cloud case (a), the flux at the depth of the absorption feature varies more with cloud coverage than compared to the $2.2 \mathrm{~km}$ case (b) because the flux at the core of the feature is determined by the amount of the absorption, namely, column-integrated $\mathrm{O}_{2}$ concentration of the species above the cloud layer. The continuum increases with increasing cloud coverage due to the higher albedo of water clouds compared to the surface reflectivity.

The right two panels of Figures 4(c), (d) are the same as Figures 4(a), (b), but with relative reflectivity. It can be seen that for the $17 \mathrm{~km}$ case (c), the relative absorption varies greatly with the cloud coverage and is deeper for the lower cloud coverage due to blocking more of the atmosphere below the cloud layer. While for the $2.2 \mathrm{~km}$ case (d), the relative reflectivity hardly varies with the cloud coverage although it is slightly shallower for the higher cloud coverage.

Our results for the modern Earth case confirm previous findings by Tinetti et al. (2006a, 2006b), Kaltenegger et al. (2007), and Kitzmann et al. (2011). We will now consider the case of earlier geological epochs in Section 3.3 and calculate the detectability of these features with a LUVOIR-sized telescope in Section 4. 

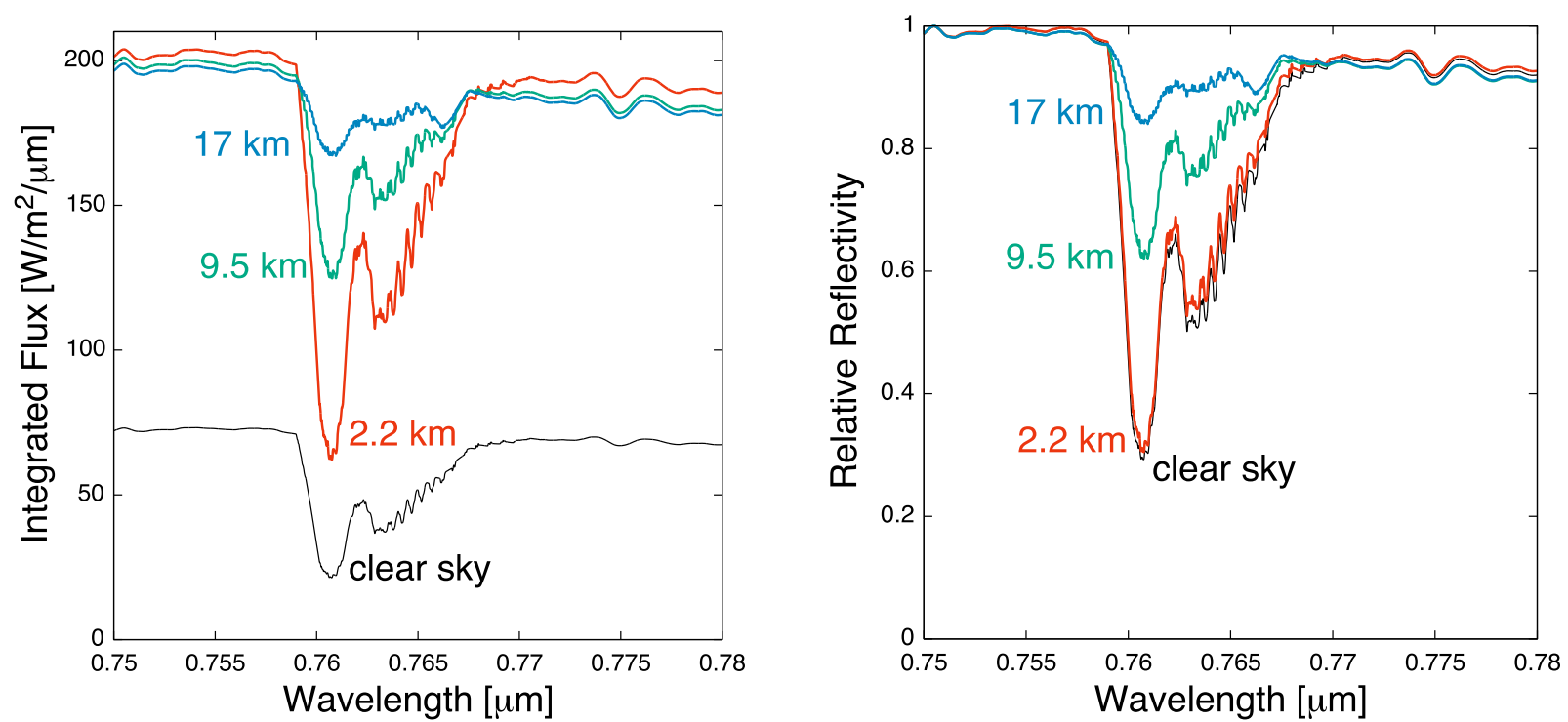

Figure 3. Same as Figure 2, with only the $\mathrm{O}_{2} A$-band feature shown in integrated flux (left) and relative reflectivity (right). The relative reflectivity is calculated by normalizing with the maximum flux between the wavelength range of $0.75-0.78 \mu \mathrm{m}$.
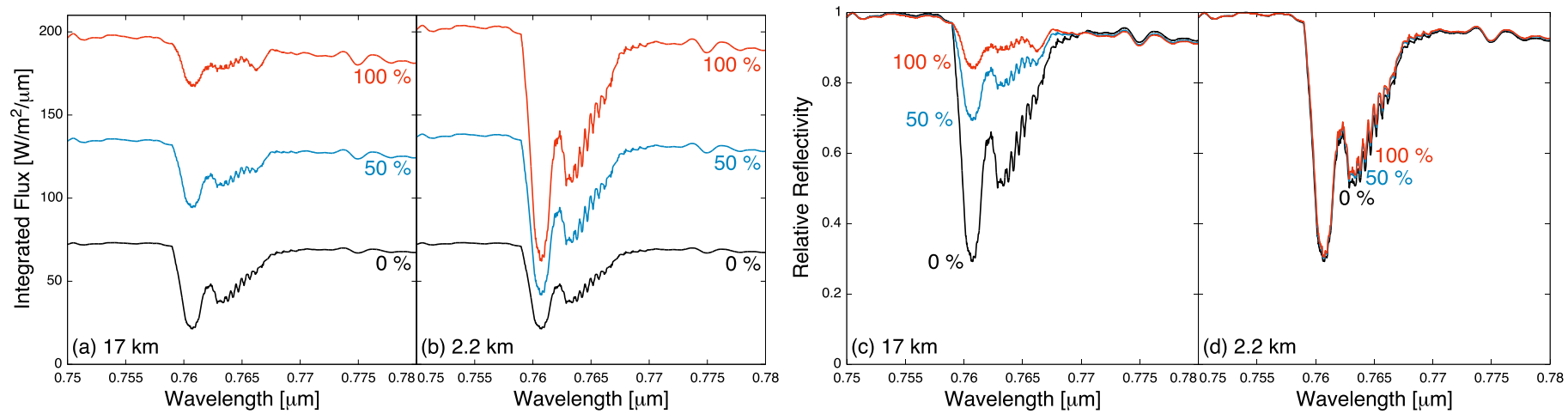

Figure 4. The $\mathrm{O}_{2} A$-band with ice cloud layers of $17 \mathrm{~km} \mathrm{(a),} \mathrm{(c)} \mathrm{and} 2.2 \mathrm{~km}$ altitude water cloud layers (b), (d) with $0 \%$ (black), 50\% (blue), and $100 \%$ (red) cloud coverage plotted with integrated flux (left two panels) and relative absorption (right two panels).

\subsection{Evolution of the Planet}

In this section, we explore the spectra of an Earth-like planet at different levels of oxygen and geological epochs. The abundance of $\mathrm{O}_{2}$ in the Earth's atmosphere has varied over time but broadly rose after two oxygenation events known as the GOE and the NOE (Lyons et al. 2014). We adopt concentrations of 0.01 PAL, 0.1 PAL, and 1.0 PAL for $2.0 \mathrm{Ga}, 0.8 \mathrm{Ga}$, and the present, respectively, where PAL stands for the present atmospheric level. Note that oxygen levels during the Proterozoic are debated and estimates range from $<0.001 \mathrm{PAL}$ to $0.4 \mathrm{PAL}$ (Canfield 2005; Kump 2008; Planavsky et al. 2014) as listed in Table 1. To explore the effect of $\mathrm{O}_{2}$ abundance on the spectra in detail, we also consider the case of $0.5 \mathrm{PAL} \mathrm{O}_{2}$ as a middle value. We calculate the spectrum model of the 0.5 PAL case using the same inputs to the radiative transfer model as modern Earth except for $\mathrm{O}_{2}$ abundance. Note this treatment is valid as long as one compares the spectrum models only around the wavelength range of $\mathrm{O}_{2}$ absorption features.

Figure 5 shows the spectrum for four different $\mathrm{O}_{2}$ abundance models, 0.01 PAL (2.0 Ga, purple), 0.1 PAL ( $0.8 \mathrm{Ga}$, light blue), 0.5 PAL (green), and 1.0 PAL (0.0 Ga, orange) assuming $60 \%$ cloud coverage with a $2.2 \mathrm{~km}$ water cloud layer. As expected, the absorption feature is deeper for larger $\mathrm{O}_{2}$ abundance.

\section{Results: Detectability of $\mathrm{O}_{2}, \mathrm{H}_{2} \mathrm{O}$, and $\mathrm{CH}_{4}$ with $L U$ VOIR}

In this section, we explore the detectability of the features of astrobiologically important gaseous molecules in the visible and near-infrared region of the Earth-like spectrum, namely, $\mathrm{O}_{2}, \mathrm{H}_{2} \mathrm{O}$, and $\mathrm{CH}_{4}$ with the proposed space telescope LUVOIR.

Figure 6 shows the modern Earth-like spectra of a clear sky atmosphere (black) and the same atmosphere with a $100 \%$ water cloud coverage layer at $2.2 \mathrm{~km}$ (red) along with $1 \sigma$ observational errors for $10 \mathrm{hr}$ observation with a LUVOIR-sized telescope calculated with the noise model. The assumed distance to the planetary system is $5 \mathrm{pc}$. Note the negative flux means that the measurement is consistent with zero flux as a Sun-like star has low flux in the NIR.

\section{1. $\mathrm{O}_{2}$ Feature}

Figure 7 shows spectrum models for the Earth-like atmosphere with $60 \%$ cloud coverage at different altitudes, $17 \mathrm{~km}$ (blue), $9.5 \mathrm{~km}$ (green), and $2.2 \mathrm{~km}$ (red) around the $\mathrm{O}_{2}$ $A$-band feature with $1 \sigma$ observational errors for $10 \mathrm{hr}$ observation calculated with the noise model for four different $\mathrm{O}_{2}$ abundances, 0.01 PAL (a), 0.1 PAL (b), 0.5 PAL (c), and 1.0 PAL (d). Water clouds are assumed for the cases of 9.5 and $2.2 \mathrm{~km}$ cloud layers of 0.01 and $0.1 \mathrm{PAL} \mathrm{O}_{2}$ abundances 

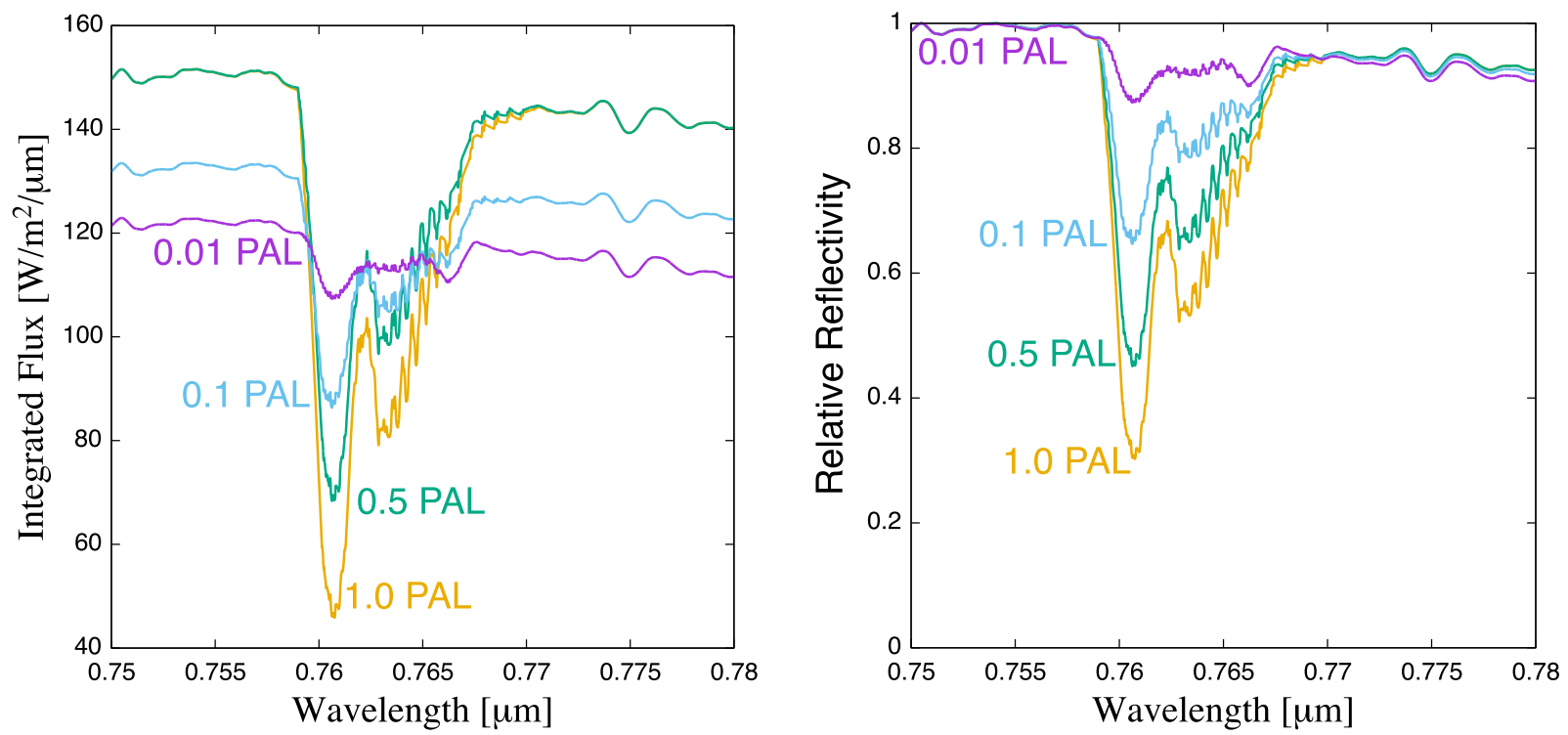

Figure 5. Integrated flux (left) and relative reflectivity (right) spectral models for four different $\mathrm{O}_{2}$ abundances, $0.01 \mathrm{PAL}$ (2.0 Ga, purple), $0.1 \mathrm{PAL}(0.8 \mathrm{Ga}$, light blue), 0.5 PAL (green), and 1.0 PAL (modern, orange) assuming a $2.2 \mathrm{~km}$ water cloud layer altitude with $60 \%$ cloud coverage.

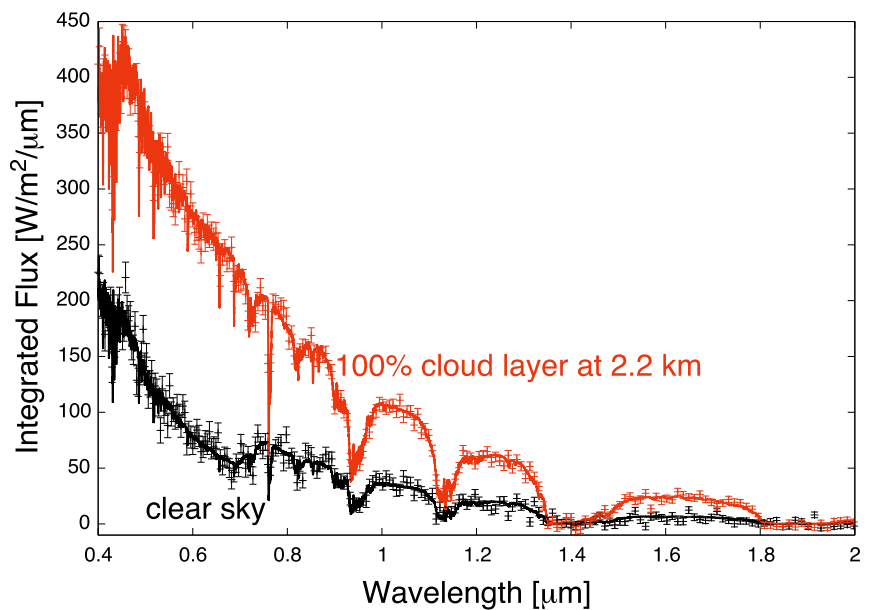

Figure 6. Modern Earth-like spectra of a clear sky atmosphere (black) and the same atmosphere with a $100 \%$ water cloud coverage layer at $2.2 \mathrm{~km}$ (red) along with $1 \sigma$ observational errors for $10 \mathrm{hr}$ observation with a LUVOIR-sized telescope calculated with the noise model. The assumed distance to the planetary system is $5 \mathrm{pc}$.

and $2.2 \mathrm{~km}$ cloud layers of 0.5 and $1.0 \mathrm{PAL} \mathrm{O}_{2}$ abundances, while ice clouds are assumed for the other cases. The assumed distance to the planetary system is $5 \mathrm{pc}$.

Again, note that the difference of the reflectivity of water and ice clouds in this wavelength region is minimal. We also note that we present the results on the grids we run the simulations and the stark contour lines come from our low-resolution grids.

We find that the observational $1 \sigma$ error bars are much larger than the $\mathrm{O}_{2}$ absorption feature depth for the 0.01 PAL $\mathrm{O}_{2}$ concentration case (a), but comparable or smaller for larger $\mathrm{O}_{2}$ concentration cases of 0.1 PAL (b), 0.5 PAL (c), and 1.0 PAL (d), especially for the cases of cloud layers at the lower altitudes. The integration time required to detect the $\mathrm{O}_{2} A$-band feature with the proposed LUVOIR telescope with $\mathrm{S} / \mathrm{N}=5$ for the $2.2 \mathrm{~km}$ altitude cloud layer and $0.5 \mathrm{PAL} \mathrm{O}_{2}$ concentration case (red line in Figure 7(c)) is $9.4 \mathrm{hr}$, almost the same as the assumed observation time. Here, we assume the wavelength region of the feature is
$0.759-0.769 \mu \mathrm{m}$. The detection time for each case in Figure 7 is tabulated in Table 3.

Figure 8 shows an intensity plot for the integration time required to detect the $\mathrm{O}_{2} A$-band feature with $\mathrm{S} / \mathrm{N}=5$ for four different $\mathrm{O}_{2}$ abundances, 0.01 PAL (a), 0.1 PAL (b), 0.5 PAL (c), and 1.0 PAL (d) with varying cloud altitude and coverage. The assumed wavelength region for the feature is $0.759-0.769 \mu \mathrm{m}$. For high-altitude ( $210 \mathrm{~km}$ for the modern case) clouds, a lower cloud coverage makes the feature deeper and the detection easier despite the smaller continuum flux for a lower cloud coverage (see Figures 4(a), (c)). For low-altitude $(\lesssim 10 \mathrm{~km}$ for the modern case) clouds, a higher cloud coverage increases the flux at the continuum, while almost the same relative depth of the feature regardless of the cloud coverage, and thus makes the detection easier for a higher cloud coverage (see Figures 4(b), (d)).

As seen in Figure 8, for the proposed LUVOIR telescope the integration time needed to detect the $\mathrm{O}_{2} A$-band feature for an Earth-like atmosphere with an $\mathrm{O}_{2}$ abundance of 0.01 PAL (a) will take typically more than $1000 \mathrm{hr}$ for the majority of the cloud parameters. The best-case scenario would be for a widespread low layer cloud, which would then make the feature detectable with $100 \mathrm{hr}$. For the 0.1 PAL, 0.5 PAL, and 1.0 PAL $\mathrm{O}_{2}$ cases, for the majority of the cloud parameter space, the detection will take approximately 100,30 , and $10 \mathrm{hr}$, respectively (see Figure 8). For the cloud parameter end cases, the minimum and maximum detection times are $10-600 \mathrm{hr}$ for the 0.1 PAL case, $3-300 \mathrm{hr}$ for the $0.5 \mathrm{PAL}$ case, and 2-200 hr for the $1 \mathrm{PAL} \mathrm{O}_{2}$ case. In particular, for the atmospheres with 0.5 and 1.0 PAL $\mathrm{O}_{2}$ abundances, the feature will be detectable with an integration time $\lesssim 10 \mathrm{hr}$ as long as there are loweraltitude $(\lesssim 8 \mathrm{~km})$ clouds with a global coverage of $\gtrsim 20 \%$. Note that modern Earth has a global cloud coverage of $\sim 50 \%-60 \%$ (Tinetti et al. 2006a; Robinson et al. 2011).

\section{2. $\mathrm{H}_{2} \mathrm{O}$ Feature}

Among the several $\mathrm{H}_{2} \mathrm{O}$ features at 0.71-0.74, 0.80-0.84, $0.90-0.98,1.1-1.2,1.3-1.5$, and $1.7-2.0 \mu \mathrm{m}$, we explore the detectability of the strongest feature at $0.90-0.98 \mu \mathrm{m}$ in this section. Figure 9 shows an intensity plot for the integration 


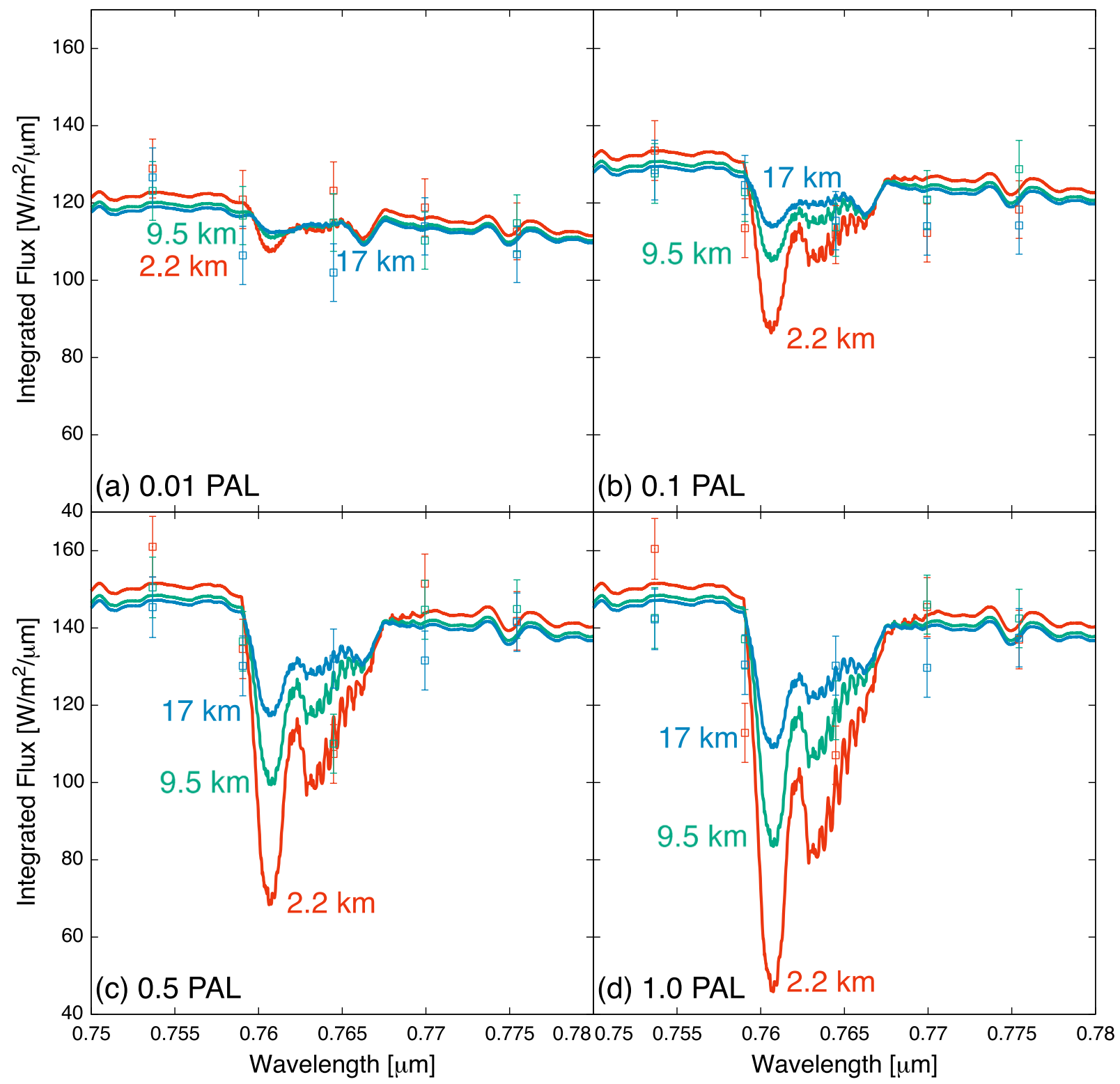

Figure 7. $\mathrm{O}_{2} A$-band feature for the Earth-like atmosphere with $60 \%$ cloud coverage at different altitudes, $17 \mathrm{~km}$ (blue), $9.5 \mathrm{~km}$ (green), and $2.2 \mathrm{~km}$ (red) with $1 \sigma$ observational errors for $10 \mathrm{hr}$ observation calculated with the noise model for four different $\mathrm{O}_{2}$ abundances, 0.01 PAL (a), 0.1 PAL (b), 0.5 PAL (c), and 1.0 PAL (d). Water clouds are assumed for the cases of 9.5 and $2.2 \mathrm{~km}$ cloud layers of 0.01 and $0.1 \mathrm{PAL} \mathrm{O}_{2}$ abundances and $2.2 \mathrm{~km}$ cloud layers of 0.5 and $1.0 \mathrm{PAL}$ $\mathrm{O}_{2}$ abundances, while ice clouds are assumed for the other cases.

Table 3

Integration Time [Hour] Required to Detect $\mathrm{O}_{2} \mathrm{~A}$-band Feature of $0.759-0.769 \mu \mathrm{m}$ in the Atmosphere of Earth-like Planet Located at $5 \mathrm{pc}$ Away with the Proposed LUVOIR Telescope with $\mathrm{S} / \mathrm{N}=5$ for the Cases of Three Different Cloud Layer Altitudes, 17, 9.5, and $2.2 \mathrm{~km}$, and Four Different $\mathrm{O}_{2}$ Abundances, 0.01, 0.1, 0.5, 1.0 PAL; the Assumed Cloud Coverage is 60\%

\begin{tabular}{lccc}
\hline \hline \multirow{2}{*}{$\mathrm{O}_{2}$ Abundance } & \multicolumn{3}{c}{ Altitude of Cloud Layer } \\
\cline { 2 - 4 } & $17 \mathrm{~km}$ & $9.5 \mathrm{~km}$ & $2.2 \mathrm{~km}$ \\
\hline $0.01 \mathrm{PAL}$ & 4800 & 1800 & 470 \\
$0.1 \mathrm{PAL}$ & 380 & 130 & 33 \\
$0.5 \mathrm{PAL}$ & 85 & 30 & 9.4 \\
$1.0 \mathrm{PAL}$ & 47 & 16 & 5.2 \\
\hline
\end{tabular}

time required to detect the $\mathrm{H}_{2} \mathrm{O}$ feature of $0.900-0.980 \mu \mathrm{m}$ with $\mathrm{S} / \mathrm{N}=5$ for three different Earth-trajectory epochs, 2.0 Ga (a), $0.8 \mathrm{Ga}(\mathrm{b})$, and the modern Earth (c) with varying cloud altitude and coverage. Same as the $\mathrm{O}_{2}$ case, for high-altitude ( $\gtrsim 3 \mathrm{~km}$ for the modern case) clouds, a lower cloud coverage makes the feature deeper and the detection easier, while for low-altitude $(\lesssim 3 \mathrm{~km}$ for the modern case) clouds, a high cloud coverage makes the detection easier due to the increased flux at the continuum. While the water clouds also have absorption at this wavelength, the ice clouds do not have such absorption and thus their relatively reflective properties basically make the required integration time slightly smaller.

Compared to the $\mathrm{O}_{2}$ case (Section 4.1), the detection time for the $\mathrm{H}_{2} \mathrm{O}$ feature significantly depends on the cloud properties, namely, altitude and coverage. Except for the extreme case of higher coverage ( $\gtrsim 80 \%$ for the modern case) clouds at high altitudes ( $\gtrsim 6 \mathrm{~km}$ for the modern case), for all the three epochs, the detection of the $\mathrm{H}_{2} \mathrm{O}$ feature will take approximately $3-10 \mathrm{hr}$, an order of magnitude smaller than that for the $\mathrm{O}_{2}$ feature. For the extreme cases, the minimum and maximum detection times are $(0.4-6) \times 10^{4} \mathrm{hr}$ for the $2.0 \mathrm{Ga}$ case, 


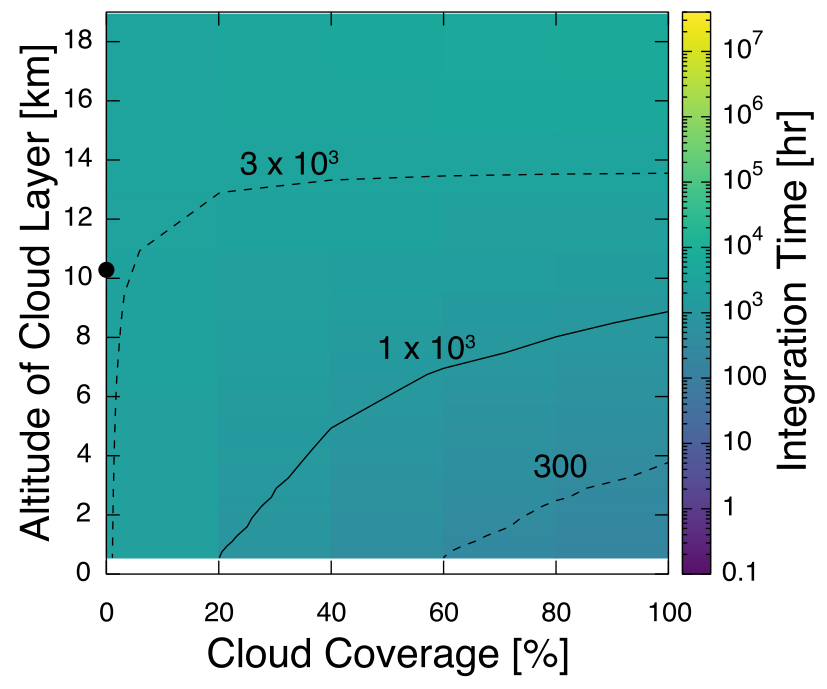

(a) 0.01 PAL $(2.0 \mathrm{Ga})$

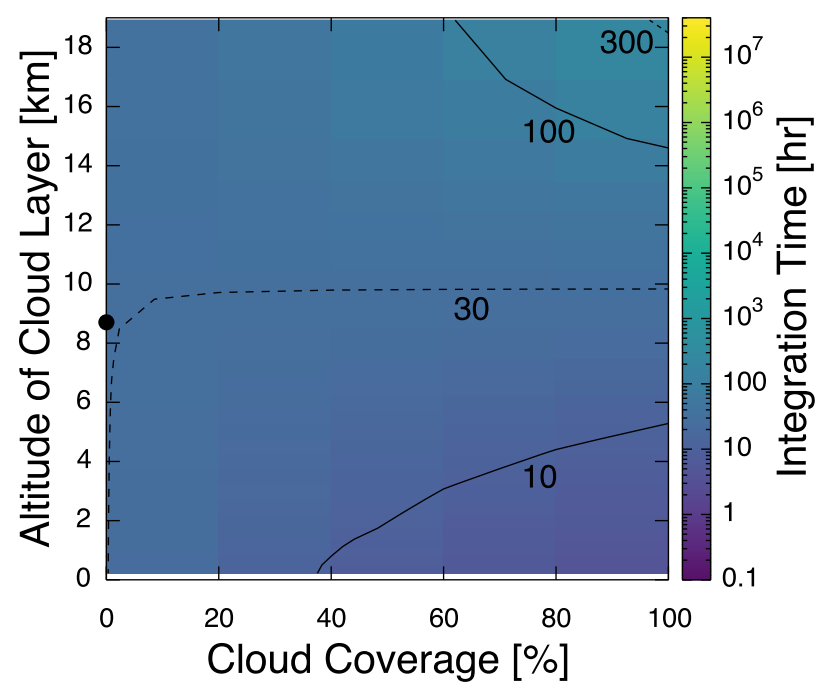

(c) $0.5 \mathrm{PAL}$

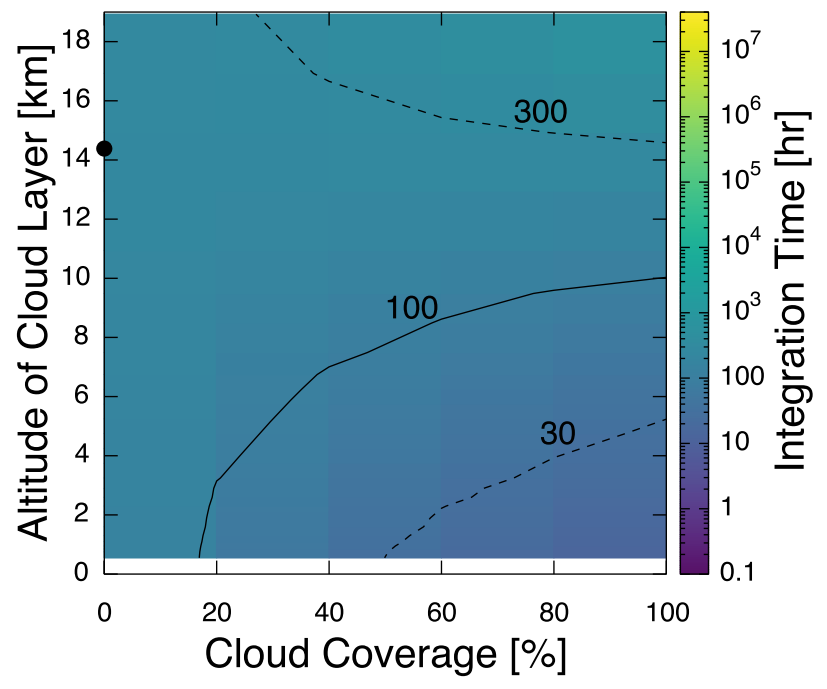

(b) 0.1 PAL $(0.8 \mathrm{Ga})$

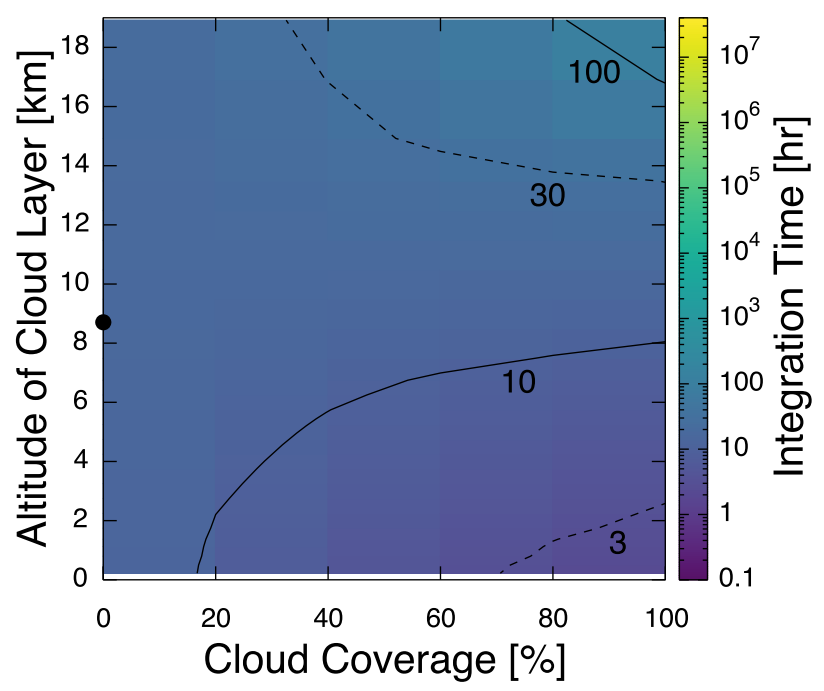

(d) 1.0 PAL (modern)

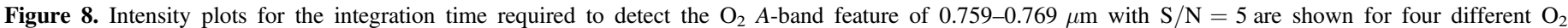

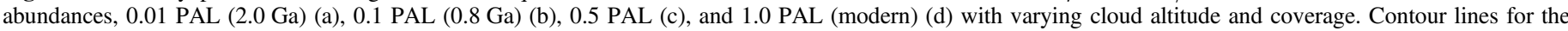

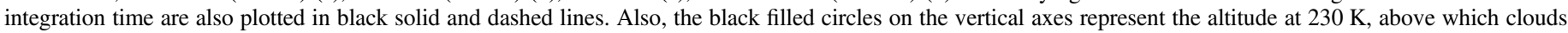
are assumed as ice ones.

$(0.2-9) \times 10^{3} \mathrm{hr}$ for the $0.8 \mathrm{Ga}$ case, and $(0.4-3) \times 10^{5} \mathrm{hr}$ for the modern case (see Figure 9). The very large detection times for the high-altitude and high cloud coverage case is due to $\mathrm{H}_{2} \mathrm{O}$ being less abundant in the upper atmosphere for planets with a cold trap, whereas $\mathrm{O}_{2}$ is well mixed.

Note the water abundance for the two earlier geological epochs in our models is largely determined by increased evaporation due to higher surface temperatures from a larger greenhouse effect despite a lower solar luminosity and is not constrained by geological proxies.

\section{3. $\mathrm{CH}_{4}$ Feature}

Figure 10 shows an intensity plot for the integration time required to detect the strongest reflected light $\mathrm{NIR} \mathrm{CH}_{4}$ feature at $1.64-1.78 \mu \mathrm{m}$ with $\mathrm{S} / \mathrm{N}=5$ for three different Earthtrajectory epochs, $2.0 \mathrm{Ga}(\mathrm{a}), 0.8 \mathrm{Ga}(\mathrm{b})$, and modern Earth (c) with varying cloud altitude and coverage.
Contrary to the cases of $\mathrm{O}_{2}$ and $\mathrm{H}_{2} \mathrm{O}$, the optical properties of water and ice clouds are quite different in this wavelength region with much higher reflectivity for water clouds, and this causes the changes of the trend at the threshold altitudes. For the water cloud region at the lower altitudes, the lower the altitude of the cloud layer becomes, and the higher the cloud coverage becomes, the detection time becomes smaller. This is because of the larger column-integrated concentration of the species above the cloud layer and the relatively reflective properties of water clouds compared to the surface. However, in the ice cloud region at the higher altitudes, the lower the altitude of the cloud layer becomes, and the lower the cloud coverage becomes, the detection time becomes smaller. This is due to the larger column-integrated concentration of the species above the cloud layer and the relatively absorbing properties of ice clouds compared to the surface in this wavelength range.

The detection of the $\mathrm{CH}_{4}$ feature will take approximately 10 and $30 \mathrm{hr}$ for the 2.0 and $0.8 \mathrm{Ga}$ cases, respectively. For the 


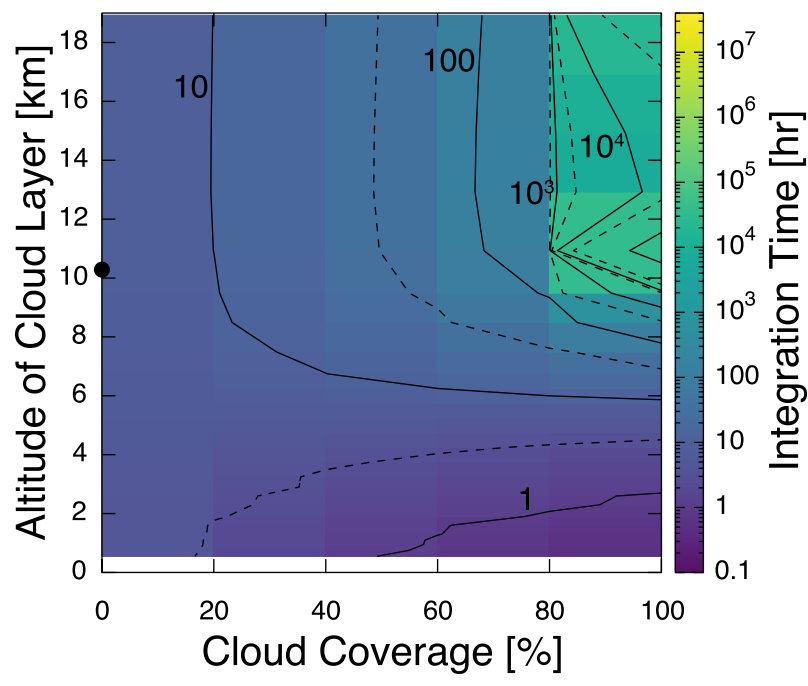

(a) $2.0 \mathrm{Ga}$

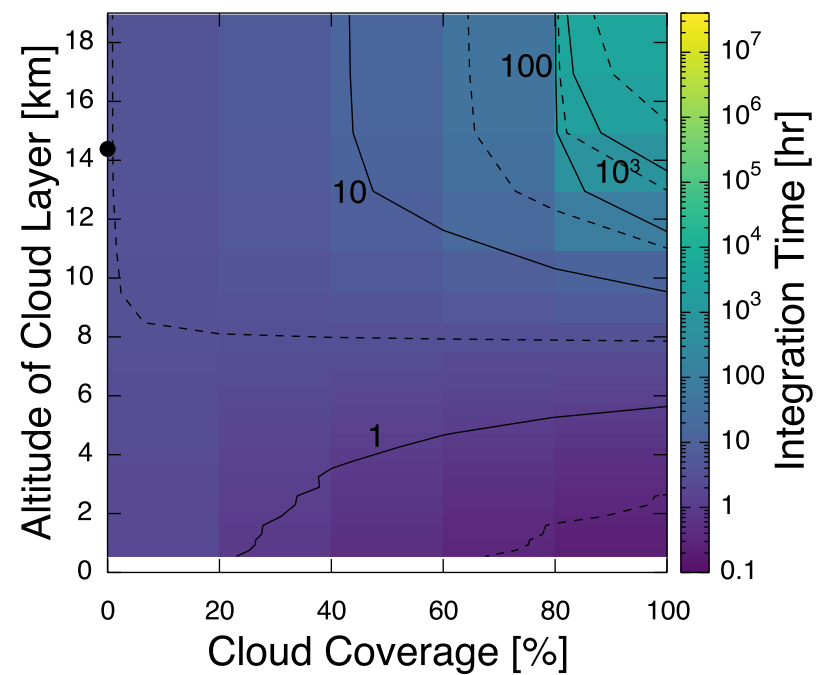

(b) $0.8 \mathrm{Ga}$

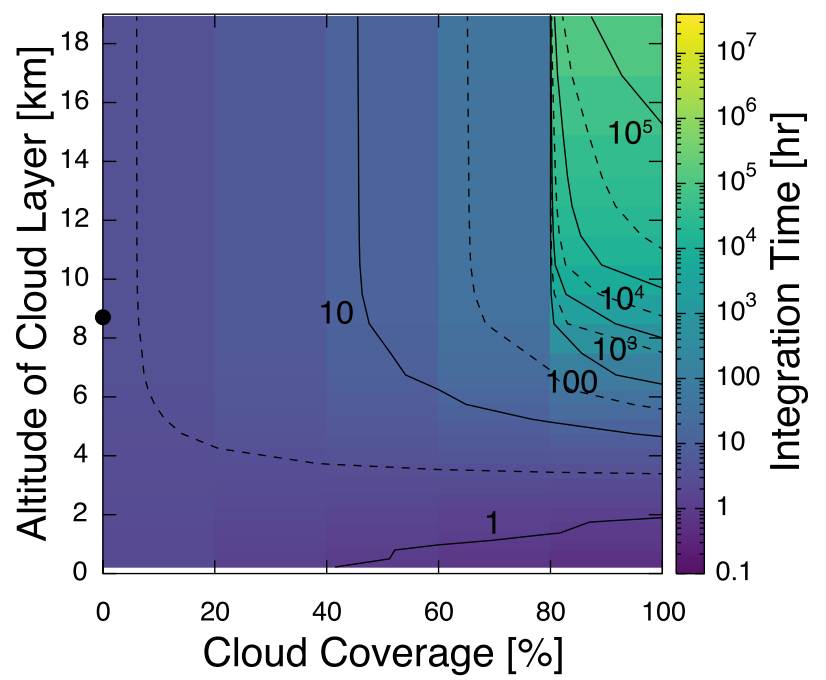

(c) Modern

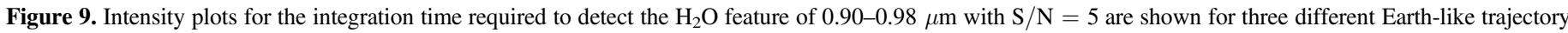

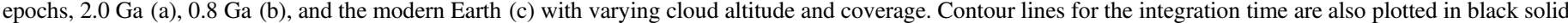

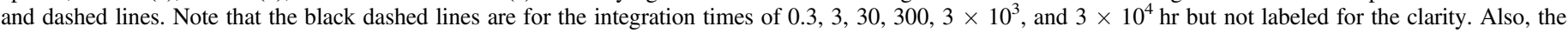
black filled circles on the vertical axes represent the altitude at $230 \mathrm{~K}$, above which clouds are assumed as ice ones.

extreme cloud parameter cases, the minimum and maximum detection times are $1-300 \mathrm{hr}$ for the $2.0 \mathrm{Ga}$ case and 2-900 hr for the $0.8 \mathrm{Ga}$ case. Here we note that the $\mathrm{CH}_{4}$ abundances for 2.0 and $0.8 \mathrm{Ga}$ cases are not well constrained and the values we adopt may be optimistic (e.g., Reinhard et al. 2017).

For the modern Earth case, however, it will take more than $6000 \mathrm{hr}$ for all the cloud parameters modeled and the feature is undetectable even with the LUVOIR-sized telescope regardless of the cloud parameters because of the relatively low $\mathrm{CH}_{4}$ abundance in the modern atmosphere and the weaker NIR feature as compared with the IR $\mathrm{CH}_{4}$ feature. For the extreme cases, the minimum and maximum detection times are 6000 to $3 \times 10^{7} \mathrm{hr}$ for the modern case (see Figure 10). The trend for the modern Earth case is the same as the 2.0 and $0.8 \mathrm{Ga}$ cases.

\section{Discussion}

In this study, we have examined the impact of cloud properties (cloud altitude and its coverage) on the detectability of the molecules on the reflectance spectra of an Earth-like planet at different geological epochs systematically. A selfconsistent microphysical model (e.g., Zsom et al. 2012; Ohno \& Okuzumi 2017, 2018; Gao \& Benneke 2018; Powell et al. 2018; Ormel \& Min 2019) would be needed to examine the plausibility of these cloud parameters, which is beyond the scope of this study. In our two-stream radiative transfer model, the cloud layer is assumed to be completely absorbing or reflective surface. In reality, however, some light can penetrate the cloud layer depending on the thickness of the cloud layer, and the cloud particle size. This effect also cannot be studied without deriving the distributions of the size and number density of the cloud particles by a microphysical cloud model and by using a multiscattering radiative transfer model.

For all the cases of detecting the $\mathrm{O}_{2} A$-band, $\mathrm{H}_{2} \mathrm{O}$ feature at $0.90-0.98 \mu \mathrm{m}$, and that of $\mathrm{CH}_{4}$ at $1.64-1.78 \mu \mathrm{m}$, we have found that the shortest integration times are for a highcoverage, low-altitude cloud layer due to the deeper absorption 


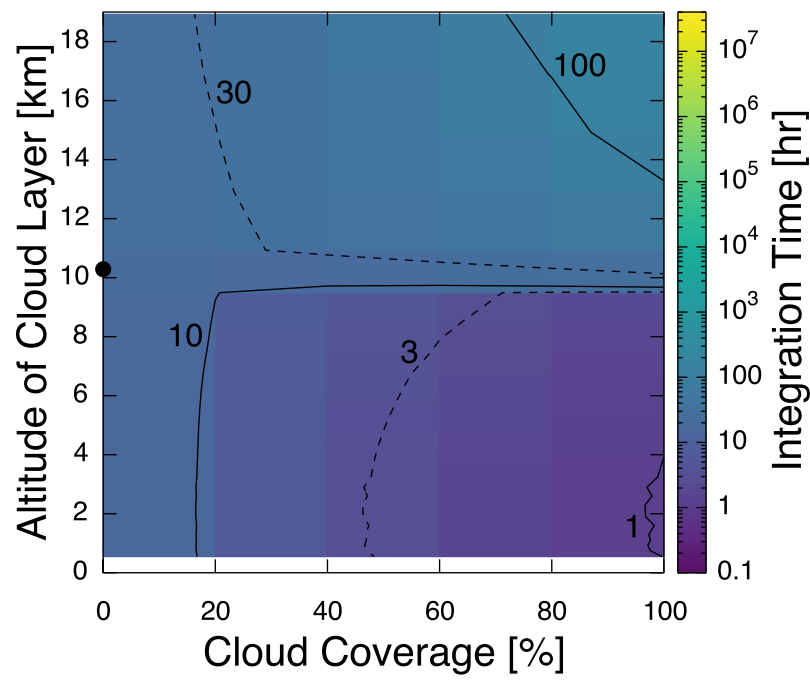

(a) $2.0 \mathrm{Ga}$

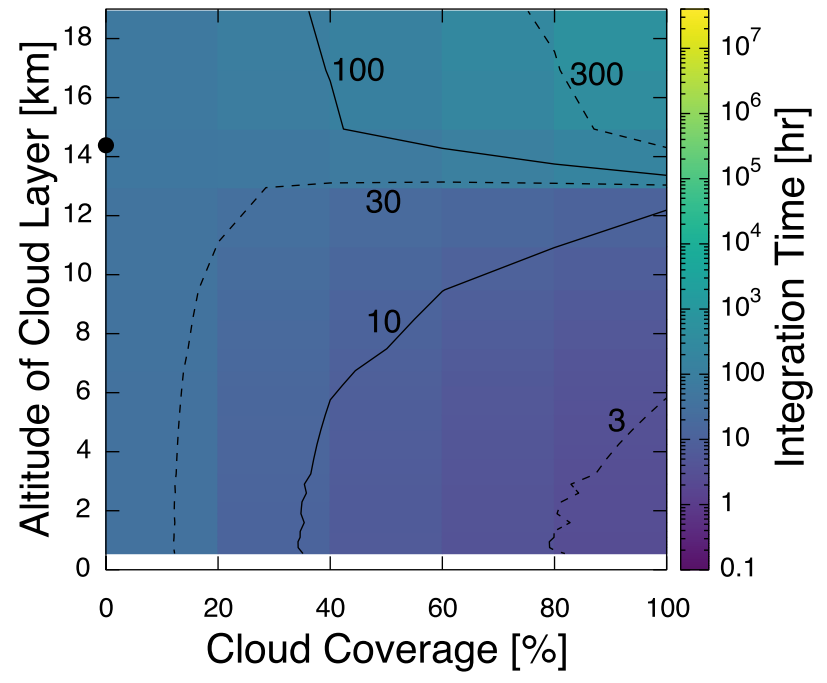

(b) $0.8 \mathrm{Ga}$

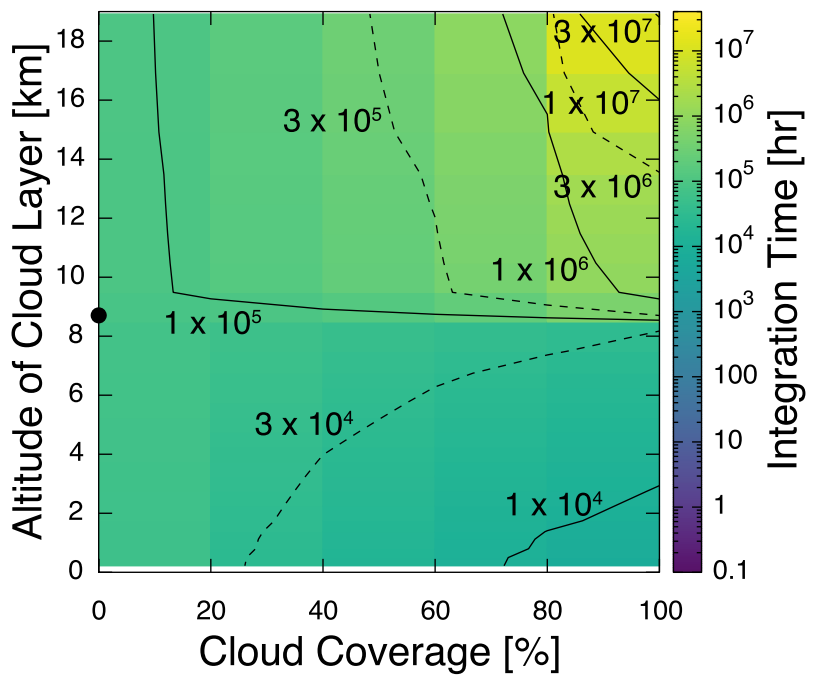

(c) Modern

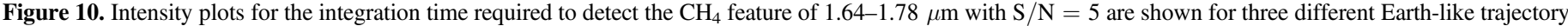

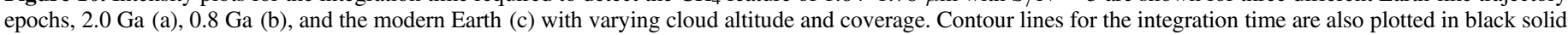
and dashed lines. Also, the black filled circles on the vertical axes represent the altitude at $230 \mathrm{~K}$, above which clouds are assumed as ice ones.

created with increased back-scattered light of the higher albedo cloud layer when compared with the surface albedo and the larger integrated column density. It is possible that this same effect on the detectability could be seen on a snowball planet (e.g., Tajika 2008; Kadoya \& Tajika 2014, 2015, 2016). While the colder temperatures may slow down bioproductivity, Earth has been through several snowball states with global glaciation well after oxygen has been a major atmospheric constituent and even quite recent in its history (see Kirschvink 1992; Hoffman et al. 2017, and references therein). These snowball states may make these features easier to detect as long as there are appreciable levels of these species in the atmosphere. As for the abundance of $\mathrm{H}_{2} \mathrm{O}$, although we have assumed larger abundances for the two earlier geological epochs, during the cooler period within the huge temporal range of temperature oscillation, it might be lower than what we have assumed, making the detection more difficult.

While we explored the detectability of specific absorption features of $\mathrm{O}_{2}, \mathrm{H}_{2} \mathrm{O}$, and $\mathrm{CH}_{4}$ via the low-resolution measurement of the flux-contrast around the wavelength range of absorption features by LUVOIR, Wang et al. (2018) investigated the detection time of $\mathrm{O}_{2}, \mathrm{H}_{2} \mathrm{O}, \mathrm{CH}_{4}$, and $\mathrm{CO}_{2}$ via a high-resolution cross-correlation technique over the wavelength range of $0.5-1.8 \mu \mathrm{m}$ by $H a b E x$ and LUVOIR. In their LUVOIR case, considering a modern Earth-like planet with a clear sky atmosphere located at 5 pc away, they reported that the required starlight suppressions for an exposure time of $100 \mathrm{hr}$ are $\sim 2 \times 10^{-9}$ and $\sim 10^{-8}$ for $\mathrm{H}_{2} \mathrm{O}$ and $\mathrm{O}_{2}$, respectively, while $\mathrm{CH}_{4}$ and $\mathrm{CO}_{2}$ are undetectable with $100 \mathrm{hr}$ exposure time.

\section{Summary}

In this study, we have explored the effect of cloud altitude and its coverage on the reflectance spectra of Earth-like planets at different geological epochs and examined the detectability of astrobiologically interesting gaseous molecules in the visible and near-infrared spectrum, namely, $\mathrm{O}_{2}, \mathrm{H}_{2} \mathrm{O}$, and $\mathrm{CH}_{4}$, by 
simulating instrumental noise for the proposed mission concept LUVOIR.

Considering an Earth-like planet located at 5 pc away, we have found that for the proposed LUVOIR telescope, the detection of the $\mathrm{O}_{2} A$-band feature $(0.76 \mu \mathrm{m})$ will take approximately 100,30 , and $10 \mathrm{hr}$ for the majority of the cloud parameters modeled for atmospheres with $0.1,0.5$, and 1.0 PAL $\mathrm{O}_{2}$ abundances, respectively. In particular, for 0.5 and 1.0 PAL $\mathrm{O}_{2}$ cases, the feature could be detectable with integration times $\lesssim 10 \mathrm{hr}$ as long as there are lower-altitude $(\lesssim 8 \mathrm{~km})$ clouds with a global coverage of $\gtrsim 20 \%$. For the $0.01 \mathrm{PAL} \mathrm{O}_{2}$ case, however, it will take more than $100 \mathrm{hr}$ for all the cloud parameters modeled.

The combined detection of $\mathrm{O}_{2}$ and $\mathrm{CH}_{4}$ remains the strongest biosignature. There is currently no known abiotic oxygen production mechanism that would persist with a simultaneously detectable amount of $\mathrm{CH}_{4}$ present. For $\mathrm{CH}_{4}$, we have found that the detection of its NIR feature at $1.64-1.78 \mu \mathrm{m}$ will take approximately 10 and $30 \mathrm{hr}$ for the $2.0 \mathrm{Ga}$ and $0.8 \mathrm{Ga}$ cases, respectively.

For the modern Earth case, however, it will take more than $6000 \mathrm{hr}$ for all the cloud parameters modeled, and the feature is undetectable even with the LUVOIR-sized telescope in its NIR feature at $1.64-1.78 \mu \mathrm{m}$ because of the relatively low $\mathrm{CH}_{4}$ abundance in the modern atmosphere.

While $\mathrm{H}_{2} \mathrm{O}$ is not a biosignature, it is an important indicator of habitability and provides necessary context for interpreting future exoplanet observations. For the $\mathrm{H}_{2} \mathrm{O}$ feature at $0.90-0.98 \mu \mathrm{m}$, we have found that except for the extreme case of higher cloud coverage at high altitudes, the detection of its strongest feature will take approximately 3-10 hr.

In summary, a LUVOIR-sized mission with a coronagraph could detect the reflected light of $\mathrm{O}_{2}, \mathrm{CH}_{4}$, and $\mathrm{H}_{2} \mathrm{O}$ for many cases comparable to Earth's geological history with a wide range of cloud parameters. To detect the combination of these gases with less than $100 \mathrm{hr}$ of observation time, however, will require more $\mathrm{CH}_{4}$ than in the modern Earth's atmosphere, $\mathrm{O}_{2}$ levels around 0.1 PAL or greater, and clouds that are lower in altitude or patchy in coverage.

We thank Tyler D. Robinson for kindly providing his noise calculation model and giving us advice and comments. We are grateful to Mark Claire for providing helpful comments on the geological constraints on the past oxygen and methane abundances. We would like to thank the Kavli Summer Program in Astrophysics 2016; Exoplanetary Atmospheres for providing us the opportunity to conduct this research and Kavli Foundation for supporting the program. We also thank the anonymous referee for careful reading and constructive comments, which helped us improve this paper greatly. Y.K. is supported by the Grant-in-Aid for JSPS Fellow (JSPS KAKENHI No. 15J08463), Leading Graduate Course for Frontiers of Mathematical Sciences and Physics, Grant-in-Aid for Scientific Research (A) (JSPS KAKENHI No. 15H02065), and the European Union's Horizon 2020 Research and Innovation Programme under grant Agreement 776403. This work was also supported by a grant from the Simons Foundation (SCOL award 339489 to S.R.) and benefited from the Exoplanet Summer Program in the Other Worlds Laboratory (OWL) at the University of California, Santa Cruz, a program funded by the Heising-Simons Foundation.

\section{ORCID iDs}

Yui Kawashima (1) https://orcid.org/0000-0003-3800-7518

\section{References}

Abe, Y., Abe-Ouchi, A., Sleep, N. H., \& Zahnle, K. J. 2011, AsBio, 11, 443 Abe, Y., Numaguti, A., Komatsu, G., \& Kobayashi, Y. 2005, Icar, 178, 27 Anglada-Escudé, G., Amado, P. J., Barnes, J., et al. 2016, Natur, 536, 437 Baldridge, A., Hook, S., Grove, C., \& Rivera, G. 2009, RSEnv, 113, 711 Canfield, D. E. 2005, AREPS, 33, 1

Claire, M. W., Catling, D. C., \& Zahnle, K. J. 2006, Geobiology, 4, 239

Claire, M. W., Sheets, J., Cohen, M., et al. 2012, ApJ, 757, 95

Des Marais, D. J., Harwit, M. O., Jucks, K. W., et al. 2002, AsBio, 2, 153

Dittmann, J. A., Irwin, J. M., Charbonneau, D., et al. 2017, Natur, 544, 333

Domagal-Goldman, S. D., Segura, A., Claire, M. W., Robinson, T. D., \& Meadows, V. S. 2014, ApJ, 792, 90

Feng, Y. K., Robinson, T. D., Fortney, J. J., et al. 2018, AJ, 155, 200

Gao, P., \& Benneke, B. 2018, ApJ, 863, 165

Gao, P., Hu, R., Robinson, T. D., Li, C., \& Yung, Y. L. 2015, ApJ, 806, 249 Gillon, M., Triaud, A. H. M. J., Demory, B.-O., et al. 2017, Natur, 542, 456

Haqq-Misra, J. D., Domagal-Goldman, S. D., Kasting, P. J., \& Kasting, J. F. 2008, AsBio, 8, 1127

Harman, C. E., Schwieterman, E. W., Schottelkotte, J. C., \& Kasting, J. F. 2015, ApJ, 812, 137

Hoffman, P. F., Abbot, D. S., Ashkenazy, Y., et al. 2017, SciA, 3, e1600983

Hu, R., Seager, S., \& Bains, W. 2012, ApJ, 761, 166

Kadoya, S., \& Tajika, E. 2014, ApJ, 790, 107

Kadoya, S., \& Tajika, E. 2015, ApJL, 815, L7

Kadoya, S., \& Tajika, E. 2016, ApJL, 825, L21

Kaltenegger, L., \& Traub, W. A. 2009, ApJ, 698, 519

Kaltenegger, L., Traub, W. A., \& Jucks, K. W. 2007, ApJ, 658, 598

Kasting, J. F., \& Ackerman, T. P. 1986, Sci, 234, 1383

Kawashima, Y., Hu, R., \& Ikoma, M. 2019, ApJL, 876, L5

Kawashima, Y., \& Ikoma, M. 2018, ApJ, 853, 7

Kawashima, Y., \& Ikoma, M. 2019, ApJ, in press

Kirschvink, J. L. 1992, in The Proterozoic Biosphere: A Multidisciplinary Study, ed. J. W. Schopf, C. Klein, \& D. Des Maris (Cambridge: Cambridge Univ. Press), 51

Kitzmann, D., Patzer, A. B. C., \& Rauer, H. 2013, A\&A, 557, A6

Kitzmann, D., Patzer, A. B. C., von Paris, P., Godolt, M., \& Rauer, H. 2011 , A\&A, 534, A63

Kodama, T., Genda, H., Abe, Y., \& Zahnle, K. J. 2015, ApJ, 812, 165

Kodama, T., Nitta, A., Genda, H., et al. 2018, JGRE, 123, 559

Kokaly, R. F., Clark, R. N., \& Swayze, G. A. 2017, USGS Spectral Library Version 7, U.S. Geological Survey Data Series 1035, https://pubs.er.usgs. gov/publication/ds1035

Kreidberg, L., Bean, J. L., Désert, J.-M., et al. 2014, Natur, 505, 69

Kump, L. R. 2008, Natur, 451, 277

Lederberg, J. 1965, Natur, 207, 9

Lovelock, J. E. 1965, Natur, 207, 568

Luger, R., \& Barnes, R. 2015, AsBio, 15, 119

Luo, G., Ono, S., Beukes, N. J., et al. 2016, SciA, 2, e1600134

Lyons, T. W., Reinhard, C. T., \& Planavsky, N. J. 2014, Natur, 506, 307

Meadows, V. S. 2017, AsBio, 17, 1022

Meadows, V. S., Reinhard, C. T., Arney, G. N., et al. 2018, AsBio, 18, 630

Narita, N., Enomoto, T., Masaoka, S., \& Kusakabe, N. 2015, NatSR, 5, 13977

Ohno, K., \& Okuzumi, S. 2017, ApJ, 835, 261

Ohno, K., \& Okuzumi, S. 2018, ApJ, 859, 34

Ormel, C. W., \& Min, M. 2019, A\&A, 622, A121

Pavlov, A. A., Hurtgen, M. T., Kasting, J. F., \& Arthur, M. A. 2003, Geo, 31, 87

Pavlov, A. A., \& Kasting, J. F. 2002, AsBio, 2, 27

Pavlov, A. A., Kasting, J. F., Brown, L. L., Rages, K. A., \& Freedman, R. 2000, JGR, 105, 11981

Planavsky, N. J., Reinhard, C. T., Wang, X., et al. 2014, Sci, 346, 635

Powell, D., Zhang, X., Gao, P., \& Parmentier, V. 2018, ApJ, 860, 18

Ramirez, R. M., \& Kaltenegger, L. 2014, ApJL, 797, L25

Reinhard, C. T., Olson, S. L., Schwieterman, E. W., \& Lyons, T. W. 2017, AsBio, 17, 287

Robinson, T. D., Meadows, V. S., Crisp, D., et al. 2011, AsBio, 11, 393

Robinson, T. D., Stapelfeldt, K. R., \& Marley, M. S. 2016, PASP, 128, 025003

Rugheimer, S., \& Kaltenegger, L. 2018, ApJ, 854, 19

Rugheimer, S., Kaltenegger, L., Segura, A., Linsky, J., \& Mohanty, S. 2015a, ApJ, 809, 57 
Rugheimer, S., Kaltenegger, L., Zsom, A., Segura, A., \& Sasselov, D. 2013, AsBio, 13, 251

Rugheimer, S., Segura, A., Kaltenegger, L., \& Sasselov, D. 2015b, ApJ, 806, 137

Sagan, C., Thompson, W. R., Carlson, R., Gurnett, D., \& Hord, C. 1993, Natur, 365,715

Sanromá, E., Pallé, E., \& García Munõz, A. 2013, ApJ, 766, 133

Sanromá, E., Pallé, E., Parenteau, M. N., et al. 2014, ApJ, 780, 52

Scott, A. C., \& Glasspool, I. J. 2006, PNAS, 103, 10861

Segura, A., Kasting, J. F., Meadows, V., et al. 2005, AsBio, 5, 706

Segura, A., Meadows, V. S., Kasting, J. F., Crisp, D., \& Cohen, M. 2007, A\&A, 472, 665

Sing, D. K., Fortney, J. J., Nikolov, N., et al. 2016, Natur, 529, 59
Stark, C. C., Roberge, A., Mandell, A., et al. 2015, ApJ, 808, 149

Stark, C. C., Roberge, A., Mandell, A., \& Robinson, T. D. 2014, ApJ, 795, 122 Tajika, E. 2008, ApJL, 680, L53

Tian, F., France, K., Linsky, J. L., Mauas, P. J. D., \& Vieytes, M. C. 2014, E\&PSL, 385, 22

Tinetti, G., Meadows, V. S., Crisp, D., et al. 2006a, AsBio, 6, 34

Tinetti, G., Meadows, V. S., Crisp, D., et al. 2006b, AsBio, 6, 881

Traub, W. A., \& Stier, M. T. 1976, ApOpt, 15, 364

Wang, J., Mawet, D., Hu, R., et al. 2018, JATIS, 4, 035001

Watson, A., Lovelock, J. E., \& Margulis, L. 1978, Biosystems, 10, 293

Wordsworth, R., \& Pierrehumbert, R. 2014, ApJL, 785, L20

Zsom, A., Kaltenegger, L., \& Goldblatt, C. 2012, Icar, 221, 603

Zsom, A., Seager, S., de Wit, J., \& Stamenković, V. 2013, ApJ, 778, 109 